\title{
Analysis of microbiota in stable patients with chronic obstructive pulmonary disease
}

\author{
ESTEFANIA AGUIRRE, ${ }^{1}$ ANTONIO GALIANA, ${ }^{1}$ ALEJANDRO MIRA,${ }^{2}$ RAMÓN GUARDIOLA, ${ }^{3}$ \\ LAURA SÁNCHEZ-GUILLÉN, ${ }^{1}$ EDUARDO GARCIA-PACHON,${ }^{4}$ MIGUEL SANTIBAÑEZ, ${ }^{5}$ \\ GLORIA ROYO ${ }^{1,6}$ and JUAN CARLOS RODRÍGUEZ ${ }^{3,6}$
}

${ }^{1}$ Section of Microbiology, Hospital General Universitario de Elche, Alicante; ${ }^{2}$ Centro Superior de Investigación en Salud Pública (CSISP), Valencia; ${ }^{3}$ Section of Microbiology, Hospital General Universitario de Alicante, Alicante; ${ }^{4}$ Section of Respiratory Medicine, Hospital General Universitario de Elche, Alicante;

${ }^{5}$ Universidad de Cantabria, IFIMAV, Santander; and ${ }^{6}$ Universidad Miguel Hernández, Elche, Spain

Aguirre E, Galiana A, Mira A, Guardiola R, Sánchez-Guillén L, Garcia-Pachon E, Santibañez M, Royo G, Rodríguez JC. Analysis of microbiota in stable patients with chronic obstructive pulmonary disease. APMIS 2015; 123: 427-432

To identify the bacterial diversity (microbiota) in expectorated sputum, a pyrosequencing method that investigates complex microbial communities of expectorated sputum was done in 19 stable chronic obstructive pulmonary disease patients (mean (SD) FEV1: $47(18 \%)$ of predicted value). Using conventional culture, 3 phyla and 20 bacterial genera were identified, whereas the pyrosequencing approach detected 9 phyla and 43 genera $(\mathrm{p}<0.001)$. In sputum the prevalent genera with pyrosequencing approach were Streptococcus, Actinomyces, Neisseria, Haemophilus, Rothia, Fusobacterium, Gemella, Granulicatella, Porphyromonas, Prevotella and Veillonella. Enterobacteriaceae, detected frequently in conventional culture, were not significantly detected with pyrosequencing methods. In addition, we found that important pathogens such as Haemophilus and Moraxella were detected more frequently with the new genetic procedures. The presence of Enterobacteriaceae is probably overestimated with conventional culture, whereas other difficult cultivable pathogens are underestimated. These studies open a new perspective for evaluating the role of bacterial colonization in chronic obstructive pulmonary disease pathogenesis and progression.

Key words: COPD; sputum microbiota.

Juan Carlos Rodríguez, S. Microbiology, Hospital General Universitario de Alicante, Alicante 03010, Spain. e-mail: rodriguez_juadia@gva.es

Chronic obstructive pulmonary disease (COPD), the fourth leading cause of death in the world, represents an important public health challenge that is both preventable and treatable. COPD is a major cause of chronic morbidity and mortality worldwide (http://www.goldcopd.org) (1). According to the EPI-SCAN study, The Epidemiologic Study of COPD in Spain (2), the prevalence of COPD in adults aged 40-80 years in Spain is estimated at $10.2 \%$, although it varies widely across different geographical areas. An estimated 2185764 Spanish COPD patients are present among a population of 21.4 million aged between 40 and 80 years.

Microorganisms are one of the main aetiological factors involved in exacerbations of COPD. In contrast, understanding of their role during stable phases of the disease is still incomplete, although

Received 27 March 2014. Accepted 14 December 2014 some studies have suggested that they actively contribute to chronic airway inflammation leading to the progression of COPD (3).

We performed a prospective observational study to identify the microbiota in the expectorated sputum of stable patients with COPD by pyrosequencing PCR-amplified bacterial 16S rRNA gene, a technique that provides a culture-independent analysis of all microbes in the sample. The aims of the present study were to describe the bacteria present in expectorated sputum using a pyrosequencing approach and to compare these findings with those obtained with conventional culture.

\section{METHODS}

\section{Patient selection}

Nineteen consecutive COPD patients attending the pulmonary clinic in a stable condition (at least 3 months without 
exacerbation or use of antibiotics for any other reason) capable of spontaneous expectoration were recruited. Patients with current or recurrent symptomatic ischaemic heart disease, congestive heart disease, cerebrovascular disease, dementia, lung cancer, known psychiatric illness, maintenance treatment with systemic corticosteroids (oral or parenteral), active tuberculosis, inflammatory bowel syndrome or insulin-dependent diabetes mellitus were excluded. Post-bronchodilator spirometry was performed in clinically stable patients (MasterLab Pro, Jaeger, Wiirzburg, Germany). Smoking habit (current smoking status, years of smoking, and number of packs of 20 cigarettes smoked per day) was noted. Patients who had stopped smoking for more than 6 months before recruitment were considered ex-smokers. Diagnosis and classification of COPD was established accordingly to GOLD recommendations (1).

All subjects were informed of the study objectives and gave their informed consent prior to their inclusion in the study. The research protocol was approved by the local ethics and research committees of the participating hospital.

\section{Sputum sampling}

Patients were asked to expectorate spontaneously; onethird of the good quality sample was used for quantitative bacterial culture, one-third was used for pyrosequencing and the remaining sample was kept in a biobank.

\section{Conventional microbiological studies}

For quantitative analysis (bacterial load), sputa were processed after liquefying with $\mathrm{N}$-acetylcysteine. Serial dilutions $(1 / 10,1 / 100,1 / 1000$ and $1 / 10000)$ were made and cultured at $37^{\circ} \mathrm{C}$ for $48 \mathrm{~h}$ in Columbia blood agar, chocolate agar and Mac Conkey agar (Biomerieux, Marcy l'Etoile, France). BCYE agar (Biomerieux, Marcy l'Etoile, France) cultures were incubated for 20 days at $37^{\circ} \mathrm{C}$ under humid atmosphere; all cultures were incubated under $\mathrm{CO}_{2}$ enriched atmosphere. No anaerobic cultures were performed. Bacterial isolates were identified by phenotypic methods and by partial sequencing of the $16 \mathrm{~S}$ rRNA gene. Genus-level identification was obtained by genetic methods.

\section{DNA isolation}

For isolating bacterial DNA we used $300 \mu \mathrm{L}$ of sample sputum and $100 \mu \mathrm{L}$ of lysis buffer $(10 \mathrm{mg} / \mathrm{mL}$ of lysozyme, $10 \%$ SDS, $10 \% \mathrm{CTAB}$ and $25 \mu \mathrm{L}$ of proteinase $\mathrm{K}$ ) were added to each sample, then the mixes were incubated for $1 \mathrm{~h}$ at $37{ }^{\circ} \mathrm{C}$ and $20 \mathrm{~min}$ at $56^{\circ} \mathrm{C}$. The purification of nucleic acids was made with phenol-chloroform and absolute ethanol precipitation. DNA extraction quality was verified by measuring with Nanodrop (Thermo Scientific, Pittsburg, Pennsylvania, USA) and only the samples with good quality yield were processed.

\section{Pyrosequencing}

A region measuring 525 bp, between position 8 and 533 of the $16 \mathrm{~S}$ ribosomal gene was amplified using the primers $8 \mathrm{~F}$ (5'-AGAGTTTGATCMTGGCTCAG-3') and 533R
(5'-TTACCGCGGCKGCTGGCAC-3'). This region comprises the regions of gene hyper-variability from $\mathrm{V} 1$ to $\mathrm{V} 3$ of the $16 \mathrm{~S}$ ribosomal gene used to classify bacterial species taxonomically. Each sample was assigned a unique multiplex identifier (MID). These amplicons were synthesized with the high-fidelity Extensor Long Range PCR Enzyme (Thermo Scientific). The PCR program designed was; $94{ }^{\circ} \mathrm{C}$ for $5 \mathrm{~min}$ followed by 30 cycles of: $94{ }^{\circ} \mathrm{C} 30 \mathrm{~s}$, $55{ }^{\circ} \mathrm{C} 30 \mathrm{~s}, 68^{\circ} \mathrm{C} 30 \mathrm{~s}$ and finally $7 \mathrm{~min}$ at $68^{\circ} \mathrm{C}$. Before pyrosequencing, the amplicons were purified using a Minielute PCR purification Kit (Qiagen, Valencia, CA, USA) and after that, AMPure beads (Agencourt, Brea, CA, USA). The amplicons were pyrosequenced using a Roche GS-FLX Titanium with Lib-L type microspheres (Roche, Basel, Switzerland).

\section{Statistical analysis and assignment of OTU's}

The pyrosequencing results were analysed bioinformatically using QIIME v1.6.0 software (4) to filter by quality and size the readings of the sequences obtained by the Roche 454 GS-FLX Titanium. After eliminating chimeric sequences from these readings, only sequences measuring between $250 \mathrm{bp}$ and $500 \mathrm{bp}$ with an end-trimming quality greater than 25 analysed in windows of 50 bases were included in the study.

The Operational Taxonomic Units (OTU'S) were assigned using the Ribosomal Database Project pyrosequencing pipeline (http://pyro.cme.msu.edu/) for the $16 \mathrm{~S}$ rRNA gene with a bootstrap cutoff of $80 \%$ and only the OTU's representing over $0.5 \%$ of the total sequences of each sample were considered. The tables were drawn up using the data obtained from the RDP classifier. Statistical analysis was performed using ANOVA test.

\section{RESULTS}

After filtering the sequences by quality and size and eliminating chimeric sequences, 20648 sequences passed the quality filters. A mean of 1086 sequences per sample were analysed.

The individual characteristics of the 19 patients are described in supplementary Table 1 . There were nine patients $(48 \%)$ with moderate COPD and ten patients $(52 \%)$ with severe COPD, seventeen were men and two women with a 71 years [SD 7.5] age mean. Nine patients were current smokers and ten were past smokers, with a smoking history average of 50.5 pack-years [SD 20.7].

With conventional cultures, 3 phyla and 20 bacterial genera were identified, while 9 phyla and 43 genera $(\mathrm{p}<0.001)$ were identified by pyrosequencing. On average (SD) the number of genera in sputum was 4.3 (1.0) with conventional culture and 19 (8.0) with pyrosequencing approach $(p<0.001)$. After taxonomic assignation of the sequences obtained by pyrosequencing (using the RDP classifier), the best represented phyla were Firmicutes and Proteobacteria present in 19/19 


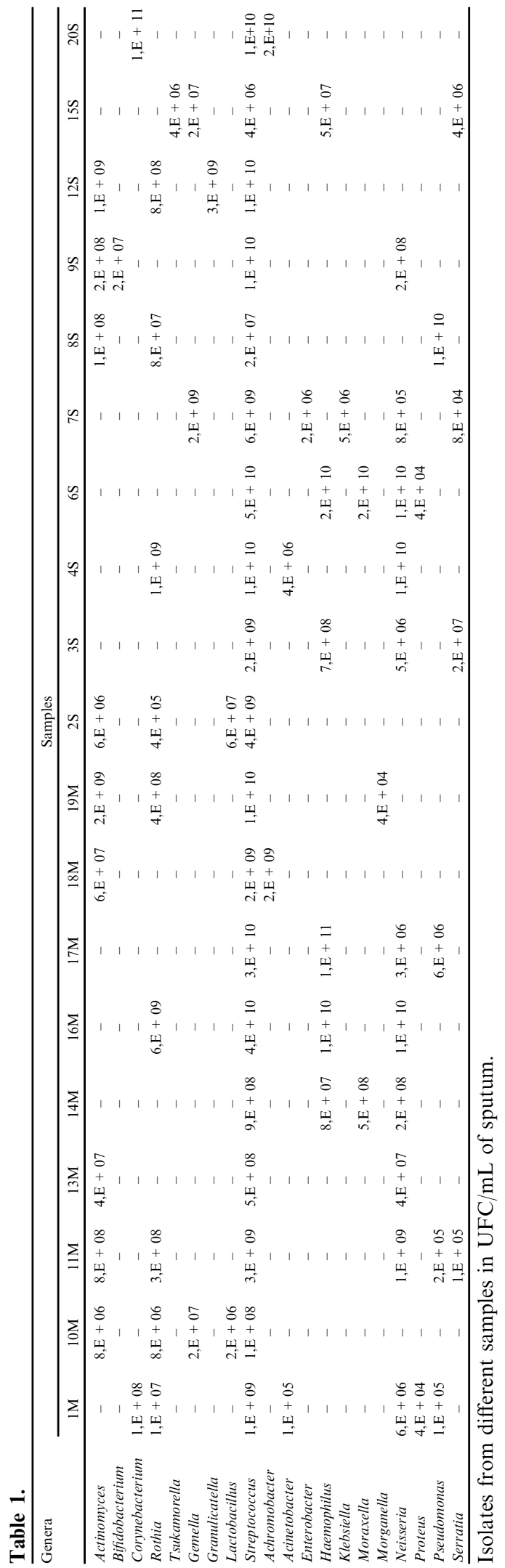

samples, followed by Actinobacteria, Bacteroidetes and Fusobacteria present in 18/19, 16/19 and 15/ 19 samples respectively. Phyla as TM7, Tenericutes, Spirochaetes and Synergistetes were found only marginally and in less than 10/19 samples. Members of Streptococcus, Fusobacterium, Prevotella, Rothia, Veillonella, Porphyromonas and Haemophilus genera dominated individual sputum samples. Other members as Haemophilus, Pseudomonas or Corynebacterium were dominant in samples $15 \mathrm{~S}$ and $17 \mathrm{M}$; $8 \mathrm{~S}$; and $20 \mathrm{~S}$ respectively. Actinomyces genus was mainly found in samples of patients from moderate group (8/9), while in samples of patients from severe group was only found in $2 / 10(\mathrm{p}<0.01)$. The distribution of the genera in the different samples is shown in Fig. 1.

Microbial culturing analysis from sputum samples showed the presence of $10^{8}$ to $10^{11} \mathrm{cfu} / \mathrm{ml}$ cultivable microorganisms per sample. The phenotypic identification and partial sequencing of the 16S rRNA gene showed the best represented phylum was Proteobacteria with 11 different genera, followed by Actinobacteria with 5 different genera and the less represented phylum was Firmicutes with 4 genera although the Streptococcus genus was present in all samples. Streptococcus and Neisseria were the most frequent genera found in 19/ 19 and 11/19 samples, respectively, followed by both Actinomyces and Rothia found in 9/19 samples. Other genera were predominant in one sample as Haemophilus (sample 17M), Pseudomonas (sample 8S) and Corynebacterium (sample 20S) (data shown in Table 1).

When comparing the two methodologies we noted that genera such as Actinomyces or Rothia were more frequently identified by the pyrosequencing assay than by conventional methods. Difficult cultivable genera as Treponema, Mycoplasma, Eikenella, Kingella or Capnocytophaga, were detected by pyrosequencing and not detected by conventional culture. Anaerobic genera as Veillonella, Prevotella, Leptotrichia, Fusobacterium, Porphyromonas, Pascardovia and Bulleidia were only detected by pyrosequencing approach due to conventional culture were carried only under aerobic conditions according to standard clinical microbiological protocols.

\section{DISCUSSION}

Sputum culture has been considered a reliable method of obtaining microbiological data (5) and, despite its limitations, remains the predominant microbiological investigation performed in the 


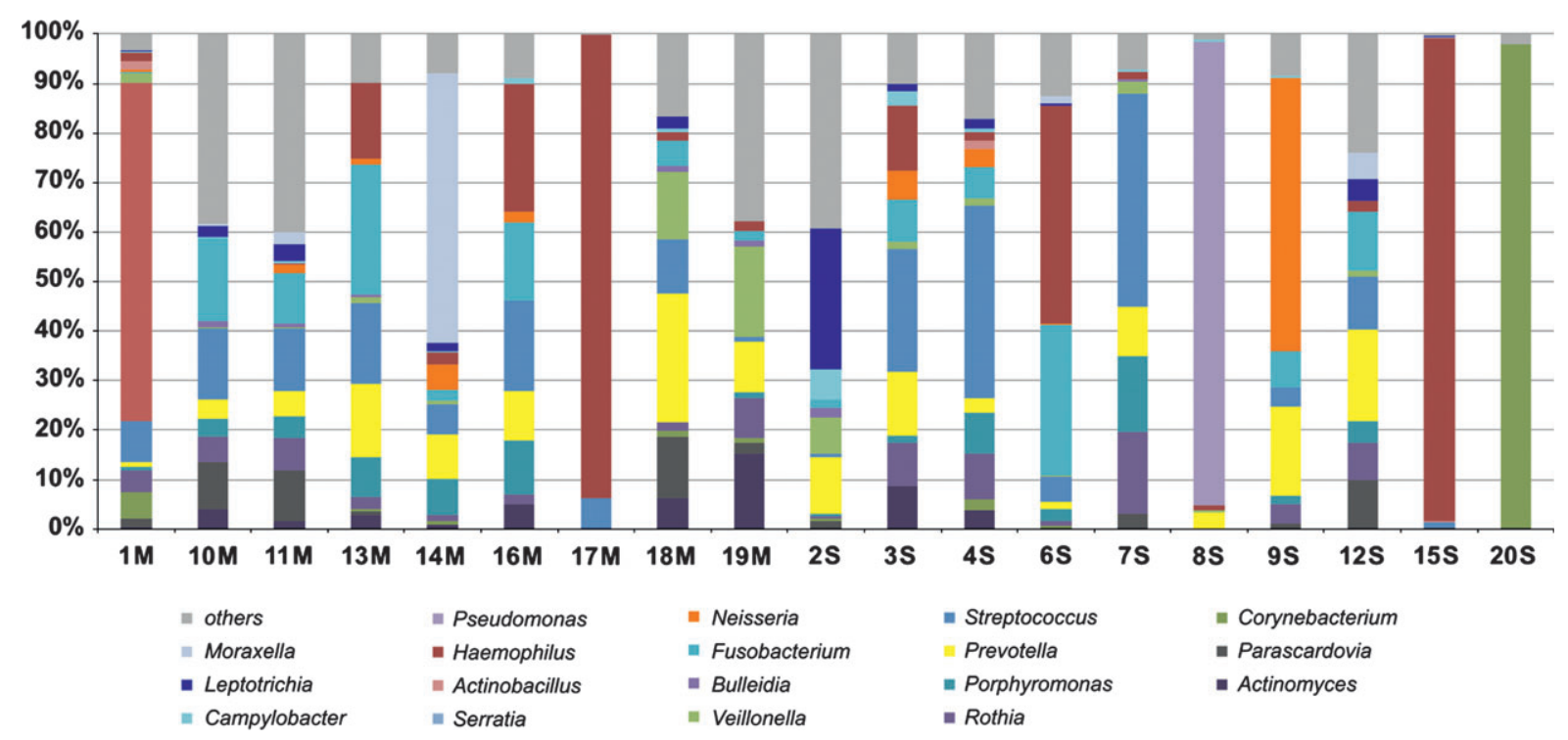

Fig. 1. Relative bacterial composition at the genus level in moderate and severe (M or S) COPD patients.

clinical setting in patients with chronic obstructive pulmonary disease (COPD) (6). Bacterial colonization in COPD has been shown to be an important factor in disease progression and exacerbations $(7,8)$.

However, conventional culture methods are not capable of isolating fastidious or emerging bacteria. It would be desirable to identify all the bacterial diversity (microbiota) in expectorated sputum so as to determine its role in inflammation, disease progression or clinical characteristics.

Thanks to recent advances, next-generation DNA-sequencing technologies have greatly enhanced capabilities for sequencing large meta-data sets (9), and provide an unprecedented opportunity to investigate the complex microbial communities associated with the human body (10). This method has been applied in several studies in sputa and other respiratory samples. In patients with asthma, microbiota composition and diversity were significantly correlated with bronchial hyperresponsiveness (11). In patients with cystic fibrosis, metagenomic studies demonstrated the presence of multiple new pathogens (12). Sze et al. (13) compared the COPD lung to the cystic fibrosis lung to demonstrate microbiome differences between these two lung diseases. These findings are also consistent with the previously published individual cultureindependent studies of the cystic fibrosis lung microbiome and COPD microbiome, not to mention consistency with older culture-based studies that highlight differences in the microbiology of these two respiratory diseases (14). Few data are available on COPD patients. Hilty et al. (15), reported some differences in the microbiota of five COPD patients compared with controls. In eight patients intubated due to COPD exacerbation, Huang et al. (16) described a great diversity of bacteria. In a study with a small sample of COPD patients, Erb-Downward et al. (17) reported limited community diversity in moderate to severe patients compared with controls.

This study using next-generation sequencing provides novel information on the microbiota of sputum in COPD patients in a stable clinical situation. As expected, metagenomics was capable of detecting a significantly higher number of bacteria than was conventional culture (Table 1 and Fig. 1). It thus reflects more accurately the wide bacterial biodiversity present in the respiratory mucosa of these patients since over $70 \%$ of the bacteria present in the respiratory mucosa cannot be cultured $(18,19)$. Our data confirm the strong evidence that culture-based methods detect only a small proportion of bacteria present in the respiratory tracts, as has been previously reported in patients with cystic fibrosis (12).

Of the seven patients in whom Enterobacteriaceae were found by conventional culture, in only one was the isolation confirmed by pyrosequencing (Table 1, Fig. 1). A similar discordance was seen when evaluating patients in whom the genus Actinomyces was present by conventional culture compared with pyrosequencing results. This is probably due to the fact that these genera are overestimated by culture even though the patients exhibit very low bacterial loads since the primers used are capable of amplifying them (20). This is of clinical interest since Enterobacteriaceae are considered to be 
important pathogens in COPD (15) and are frequently isolated in conventional culture, as in our sample. Since this may modify patterns of antibiotic treatment, further studies may explain the role of this genus in COPD patients.

On the other hand, pathogens such as Haemophilus and Moraxella, whose pathogenicity in these patients has been demonstrated in multiple studies, are more often detected by molecular methods due to the difficulties involved in isolating these microorganisms using conventional culture-based methods. This higher prevalence should also be analysed in greater depth in order to optimize the antibiotic treatment normally administered empirically in these patients. With this new methodology such optimization would be possible (21).

Metagenomic methods allowed us to identify anaerobes in expectorated sputum. In our series, all patients had anaerobes, which accounted for about $15 \%$ of the bacterial load. The clinical significance of this finding needs to be established in the light of these new tools that make it possible to obtain a much better understanding of this complex group of bacteria which to date are little understood due to the serious limitations of culture-based methods for isolating these microorganisms. Very few metagenomic analysis data are available in patients with COPD. Compared with healthy individuals and using bronchoscopy samples, Hilty et al. (15) reported in five COPD patients a higher isolation of phylum Proteobacteria (including Haemophilus) and lower proportion of phylum Bacteroidetes (mainly Prevotella). Interestingly, a decrease in total bacterial load and a diminished quantity of Bacteroidetes has also been described in inflammatory bowel disease (22). It is known that Prevotella spp. inhibit the growth of other bacteria (commensal flora) that may play a role in immune response (15). In our series, Prevotella spp was present in $90 \%$ of COPD patients, but at a very low proportion (about $4 \%$ of bacterial load). Huang et al. (16) studied with metagenomic technology eight intubated COPD patients with exacerbation and reported a greater diversity of bacteria than previously appreciated in the airways of these patients.

Our sample of patients probably does not represent all COPD patients because obviously we only included those able to expectorate spontaneously. However, in a large study, the frequency of sputum expectoration was similar in all proposed subtypes of COPD (23). Nevertheless, it would be very interesting to investigate the lung microbiota with other methods (bronchoalveolar lavage, surgical samples) in patients with and without expectoration or other clinically relevant symptoms or characteristics (24).
Microbiota in stable COPD patients shows a great diversity of bacteria that differ from bacteria detected in conventional cultures. Thus, metagenomic studies open a new perspective for evaluating the role of bacterial colonization in COPD pathogenesis and progression and may contribute to a more comprehensive understanding of this serious disease $(9$, 25 ) since the lung is a complex structure composed of cells belonging to all three domains of life on Earth, Eukarya, Bacteria and Archaea, as well as their viruses. The microbiome constitutes the last human organ under active research. Like any other organ, the microbiome has its own pathophysiology, and individual health might be damaged when its collective population structure is altered (26).

This study was supported by grants 'Ayudas para la realización de trabajos de investigación en materia de atención de media y larga estancia. Agencia Valenciana de Salud. 2010' (MLE 2/10), 'Fundación de la Comunidad Valenciana para la investigación biomédica, la docencia y la cooperación internacional y para el desarrollo del Hospital General Universitario de Elche' (FIBELX-CO11/02), 'Consejeria de Sanitat. Generalitat Valenciana' (AP-168/ 11), 'Ayuda de investigación de la Fundación Bienvenida Navarro Luciano Trípodi 2010-2011 y 2011-2012' and from the Spanish Ministry of Health (Instituto Salud Carlos III PI12/02209).

\section{REFERENCES}

1. Global Initiative for Chronic Obstructive Lung Disease (GOLD). 2011 Management and Prevention of COPD. Available at: http://www.goldcopd.org/.

2. Miravitlles M, Soriano JB, Garcia-Rio F, Munoz L, Duran-Tauleria E, Sanchez G, et al. Prevalence of COPD in Spain: impact of undiagnosed COPD on quality of life and daily life activities. Thorax 2009;64:863-8.

3. Matkovic Z, Miravitlles M. Chronic bronchial infection in COPD. Is there an infective phenotype? Respir Med 2013;107:10-22.

4. Caporaso JG, Kuczynski J, Stombaugh J, Bittinger $\mathrm{K}$, Bushman FD, Costello EK, et al. QIIME allows analysis of high-throughput community sequencing data. Nat Methods 2010;7:335-6.

5. Angrill J, De Agusti C, Celis R, Raño A, Gonzalez J, Sole $\mathrm{T}$, et al. Bacterial colonisation in patients with bronchiectasis: microbiological pattern and risk factors. Thorax 2002;57:15-9.

6. Sykes A, Mallia P, Johnston SL. Diagnosis of pathogens in exacerbations of chronic obstructive pulmonary disease. Proc Am Thorac Soc 2007;4:642-6.

7. Wilkinson TMA, Patel IS, Wilks M, Donaldson GC, Wedzicha JA. Airway bacterial load and FEV1 decline in patients with chronic obstructive pulmonary disease. Am J Respir Crit Care Med 2003;167: 1090-5. 
8. Patel IS, Seemungal TAR, Wilks M, Lloyd-Owen SJ, Donaldson GC, Wedzicha JA. Relationship between bacterial colonisation and the frequency, character, and severity of COPD exacerbations. Thorax 2002;57:759-64.

9. Petrosino JF, Highlander S, Luna RA, Gibbs RA, Versalovic J. Metagenomic pyrosequencing and microbial identification. Clin Chem 2009;55:856-66.

10. Rogers GB, Bruce KD. Next-generation sequencing in the analysis of human microbiota: essential considerations for clinical application. Mol Diagn Ther 2010;14:343-50.

11. Huang YJ, Nelson CE, Brodie EL, Desantis TZ, Baek MS, Liu J, et al. Airway microbiota and bronchial hyperresponsiveness in patients with suboptimally controlled asthma. J Allergy Clin Immunol 2011;127:372-81.

12. Bittar F, Richet H, Dubus JC, Reynaud-Gaubert M, Streimer N, Sarles J, et al. Molecular detection of multiple emerging pathogens in sputa from cystic fibrosis patients. PLoS ONE 2008;3:e2908.

13. Sze MA, Dimitriu PA, Hayashi S, Elliott WM, McDonough JE, Gosselink JV, et al. The lung tissue microbiome in chronic obstructive pulmonary disease. Am J Respir Crit Care Med 2012;185:1073-80.

14. Erb-Downward JR, Huffnagle G, Martinez FJ. The Microbiota in Respiratory Disease. Am J Respir Crit Care Med 2012;185:1037-8.

15. Hilty M, Burke C, Pedro H, Cardenas P, Bush A, Bossley C, et al. Disordered microbial communities in asthmatic airways. PLoS ONE 2010;5:e8578.

16. Huang YJ, Kim E, Cox MJ, Brodie EL, Brown R, Wiener-Kronish JP, et al. A persistent and diverse airway microbiota present during chronic obstructive pulmonary disease exacerbations. OMICS 2010;14:9-17.

17. Erb-Downward JR, Thomson DL, Han MK, Freeman CM, McCloskey L, Schmidt LA, et al. Analysis of the lung microbiome in the 'healthy' smoker and in COPD. PLoS ONE 2011;6:2.

18. Williamson SJ, Yooseph S. From bacterial to microbial ecosystems (metagenomics). Methods Mol Biol 2012;804:35-55

19. Han MK, Huang YJ, Lipuma JJ, Boushey HA, Boucher RC, Cookson WO, et al. Significance of the microbiome in obstructive lung disease. Thorax 2012;67:456-63.

20. Bailey MT, Dowd SE, Parry NM, Galley JD, Schauer DB, Lyte M. Stressor exposure disrupts commensally microbial populations in the intestines and leads to increased colonization by Citrobacter rodentium. Infect Immun 2010;78:1509-19.

21. Sialer S, Adamantia L, Guerrero M, Torres A. Relation between chronic obstructive pulmonary disease and antibiotics. Curr Infect Dis Resp 2012;14: $300-7$.

22. Frank DN, StAmand AL, Feldman RA, Boedeker EC, Harpaz N, Pace NR. Molecular-phylogenetic characterization of microbial community imbalances in human inflammatory bowel diseases. Proc Natl Acad Sci USA 2007;104:13780-5.

23. Garcia-Aymerich J, Gomez FP, Benet M, Farrero E, Basagaña X, Gayete A, et al. PAC-COPD Study Group. Identification and prospective validation of clinically relevant chronic obstructive pulmonary disease (COPD) subtypes. Thorax 2011;66:430-7.

24. Cabrera-Rubio R, Garcia-Núñez M, Setó L, Antó JM, Moya A, Monsó E, et al. Microbiome diversity in the bronchial tracts of patients with chronic obstructive pulmonary disease. J Clin Microbiol 2012; 50:3562-8.

25. Soriano JB, Brusasco V, Dinh-Xuan AT. The European Respiratory Journal makes COPD a priority. Eur Respir J 2011;38:999-1001.

26. Human Microbiome Project Consortium. Structure, function and diversity of the healthy human microbiome. Nature 2012;486:207-14.

\section{SUPPORTING INFORMATION}

Additional Supporting Information may be found in the online version of this article:

Table S1. Characteristics of patients as a function of COPD status. 